\title{
On impurity handling in High Performance Stellarator/Heliotron plasmas
}

\author{
R Burhenn ${ }^{1}$, Y Feng ${ }^{1}$, K Ida ${ }^{2}$, H Maassberg ${ }^{1}$, K J McCarthy ${ }^{3}$, D Kalinina ${ }^{2}$, \\ M Kobayashi $^{2}$, S Morita ${ }^{2}$, Y Nakamura ${ }^{2}$, H. Nozato ${ }^{4}$, S Okamura ${ }^{2}$, S Sudo ${ }^{2}$, \\ C Suzuki $^{2}$, N Tamura ${ }^{2}$, A Weller ${ }^{1}$, M Yoshinuma ${ }^{2}$, B Zurro ${ }^{3}$ \\ E-mail: rainer.burhenn@ipp.mpg.de \\ ${ }^{1}$ Max-Planck-Institute für Plasmaphysik, EURATOM Association, D-17491 \\ Greifswald, Germany \\ ${ }^{2}$ National Institute for Fusion Science, 322-6 Oroshi-cho, Toki-shi, Gifu-ken 509- \\ 5292, Japan \\ ${ }^{3}$ Laboratorio National de Fusion, Asociacion EURATOM-CIEMAT, E-28040 \\ Madrid, Spain \\ ${ }^{4}$ National Metrology Institute of Japan, National Institute of Advanced Industrial \\ Science and Technology, Tsukuba, Ibaraki 305-8563, Japan
}

\begin{abstract}
The Large Helical Device (LHD) and Wendelstein 7-X (W7-X, under construction) are experiments specially designed to demonstrate long pulse (quasi steady-state) operation, which is an intrinsic property of Stellarators and Heliotrons. Significant progress had been made in establishing high performance plasmas. A crucial point is the increasing impurity confinement at high density observed at several machines (TJ-II, W7-AS, LHD) which can lead to impurity accumulation and early pulse termination by radiation collapse. In addition, theoretical predictions for non-axisymmetric configurations predict the absence of impurity screening by ion temperature gradients in standard ion-root plasmas. Nevertheless, scenarios were found where impurity accumulation was successfully avoided in LHD and W7-AS due to the onset of friction forces in the (high density and low temperature) scrape-off-layer, the generation of magnetic islands at the plasma boundary and to a certain degree also by ELMs, flushing out impurities and reducing the net-impurity influx into the core. In both the W7-AS High Density H-mode (HDH) regime and in the case of application of sufficient ECRH heating power a reduction of impurity core confinement was observed. The exploration of such purification mechanisms is a demanding task for successful steady-state operation. Impurity transport at the plasma edge/SOL was identified to play a major role for the global impurity behaviour in addition to the core confinement.
\end{abstract}

Keywords: impurity transport, stellarator

Pacs: 52.25.Fi, 52.25.Vy, 52.55.Hc, 52.55.Rk

\section{Introduction}

In Stellarators and Heliotrons the plasma confining magnetic fields are exclusively produced by external coil systems without the net currents induced in the plasma column which are a fundamental part of the tokamak concept. Consequently, these devices do not suffer from current related drawbacks (disruptions, instabilities) and offer the intrinsic potential for continuous plasma 
On impurity handling in High Performance Stellarator/Heliotron plasmas

operation. The Large Helical Device (LHD) and Wendelstein 7-X (W7-X), the latter presently under construction, already comply with the technical requirements of long pulse operation (superconducting coils) and focus upon the establishment of high performance long-pulse plasmas. One critical issue is the avoidance of impurity accumulation which - under unfavourable confinement conditions for impurity ions - can lead to early pulse termination by radiation collapse. Theoretical predictions based on neoclassical transport theory for non-axisymmetric configurations underline the importance of this issue: in contrast to tokamaks where the "ion temperature screening" of impurity ions is a beneficial mechanism preventing impurity accumulation, for stellarators all thermodynamic forces are predicted to support accumulation in the standard case with negative radial electric field, the so-called ion-root regime [1]. Additionally, fears are raised by experimental indications for improved confinement at higher density [2]. Only in low-density scenarios at W7-AS and LHD is it possible to establish positive ion or electron roots with positive radial electric fields which flush out impurities. But even high pressure plasmas with high density and ion temperature simultaneously achieved within one discharge are interesting candidates for high-performance operation and might be afflicted with impurity problems. The relevance of the unfavourable predictions for helical devices finally depends on the predominantly neoclassical or turbulent character of the plasma. Nevertheless, neoclassical ion transport increases with temperature and might gain increasing importance in fusion-relevant plasmas at high temperatures. For this reason, exploration of favourable scenarios capable of preventing impurity accumulation becomes important. The establishment of proper ambipolar or driven radial electric fields and simultaneously the reduction of impurity influx at the plasma edge is required for long-pulse impurity control. The same issue is of course of relevance for helium exhaust in future reactors. In this work an inter-machine comparison of selected purification processes is presented in the frame of the International Stellarator/Heliotron Profile Database (ISHPDB) activities in order to prepare the physics basis for long-pulse operation in Stellarators/Heliotrons.

\section{General considerations on impurity transport in stellarators}

The impurity evolution in plasmas is basically determined by transport processes in three plasma regions: (a) the direct interaction zone between plasma and surface of plasma-facing components responsible for the impurity sources caused by sputtering of wall material, (b) the scrape-off layer (SOL) controlling the impurity influx across the separatrix and, consequently, to the core plasma and (c) the confinement properties of the core plasma itself including local transport barriers which might evolve under certain conditions. Each of these regions have their own relevance for the global impurity behaviour and only their simultaneous treatment enables one to assess the role of intrinsic impurity ions in fusion plasmas. However, the complexity of the underlaying physical processes requires elaborate models for each zone, so that the transport studies often have to be restricted to one of these regions and afterwards fitted together.

For a given core transport the "effective" impurity source into the core plasma, determined by the impurity source strength and the SOL transport, is directly related to the core impurity concentration and, consequently, to the total radiation level in the equilibrium state. Screening processes in the SOL as well as reduction of sputtering of high- $\mathrm{Z}$ material by shielding precautions or wall conditioning (boronization) help to minimize the radiation power loss. The core confinement of impurities finally determine the time scale for impurity exhaust.

In the tracer approximation neoclassical impurity fluxes in the bulk plasma can be expressed in 
On impurity handling in High Performance Stellarator/Heliotron plasmas

the general form

$$
\Gamma_{\alpha}=-n_{\alpha} D_{11}^{\alpha}\left\{\frac{n_{\alpha}^{\prime}}{n_{\alpha}}-q_{\alpha} \frac{E_{r}}{T_{\alpha}}+\frac{D_{12}^{\alpha}}{D_{11}^{\alpha}} \frac{T_{\alpha}^{\prime}}{T_{\alpha}}\right\}
$$

for species $\alpha=e, i, Z$ (electrons, background ions, impurity ions with ionic charge $\mathrm{q}_{\mathrm{Z}}=\mathrm{Z}$ or ionization state $\mathrm{Z}+$ ), density $\mathrm{n}_{\alpha}$, the radial electric field $\mathrm{E}_{\mathrm{r}}$ determined by the ambipolarity condition $\Gamma_{\mathrm{i}}=\Gamma_{\mathrm{e}}$, the temperature $\mathrm{T}_{\alpha}$, and the diagonal and off-diagonal diffusion coefficients $\mathrm{D}_{11}{ }^{\alpha}, \mathrm{D}_{12}{ }^{\alpha}$ of the transport matrix. In stellarators the three-dimensional topology of the magnetic field leads to different scalings of the transport with collision frequency $v(1 / v, \operatorname{sqrt}(v), v)$ in the long-mean-free-path ( $(\mathrm{mfp})$ regime and the transport coefficients depend on $\mathrm{E}_{\mathrm{r}}$. Hence, no analytical solution for $E_{r}$ exists and the ambipolarity condition of the particle fluxes determining $E_{r}$ may have multiple roots depending on the magnetic configuration and plasma parameters. For the "standard ion root" $\left(\Gamma_{\mathrm{i}}\left(\mathrm{E}_{\mathrm{r}}=0\right)>>\Gamma_{\mathrm{e}}, \mathrm{Zn}_{\mathrm{Z}}<<\mathrm{n}_{\mathrm{e}}\right)$ substitution of $\mathrm{E}_{\mathrm{r}} / \mathrm{T}_{\mathrm{Z}}$ in eq. 1 for the impurity ions using the ambipolarity condition $\Gamma_{\mathrm{i}}=\Gamma_{\mathrm{e}}$ and assuming $\Gamma_{\mathrm{i}}\left(\mathrm{E}_{\mathrm{r}}\right)=0$ and $\mathrm{T}_{\mathrm{i}}=\mathrm{T}_{\mathrm{Z}}$ reveals for the impurity transport fluxes

$$
\Gamma_{Z}=-n_{Z} D_{11}^{Z}\left\{\frac{n_{Z}^{\prime}}{n_{Z}}+\frac{D_{12}^{Z}}{D_{11}^{Z}} \frac{T_{i}^{\prime}}{T_{i}}-Z\left(\frac{n_{i}^{\prime}}{n_{i}}+\frac{D_{12}^{i}}{D_{11}^{i}} \frac{T_{i}^{\prime}}{T_{i}}\right)\right\}=-D_{Z} n_{Z}^{\prime}+n_{Z} V_{Z}
$$

In case of the ion-root the assumptions $\Gamma_{\mathrm{i}}\left(\mathrm{E}_{\mathrm{r}}\right)=0$ and $\mathrm{T}_{\mathrm{i}}=\mathrm{T}_{\mathrm{Z}}$ are appropriate for high performance plasmas because the dependence of $\mathrm{D}^{\mathrm{i}}$ on $\mathrm{E}_{\mathrm{r}}$ causes a strong reduction of the particle fluxes. At higher densities $\left(\mathrm{n}_{\mathrm{e}}>1 \times 10^{20} \mathrm{~m}^{3}\right)$ the coupling between electrons and ions is sufficiently large to achieve comparable temperatures.

In a simplified picture, $D_{Z}$ represents all coefficients connected with the impurity density gradient (diffusive part) and $\mathrm{V}_{Z}$ all convective terms related to density and temperature gradients of the background gas. In many cases neoclassical transport coefficients $D_{Z}$ and $V_{Z}$ are not sufficient to describe the experimental data. Therefore, so-called "anomalous" coefficients (originating from turbulent transport processes) have to be used in addition to fit the experimental results.

With increasing $\mathrm{Z}$ the third term inside the brackets becomes dominant and depends exclusively on the properties of the background gas. In case of peaked particle density profiles impurities are drawn towards the center. Opposite to tokamaks where the coefficient $\mathrm{D}_{12}{ }^{\mathrm{i}} / \mathrm{D}_{11}{ }^{\mathrm{i}}$ in front of the thermal gradient can be negative ("impurity screening") it is predicted to be always positive for stellarators and supports impurity accumulation in the presence of peaked temperature profiles. The situation might only change if the collisionality of the background gas changes from lmfp to the Pfirsch-Schlueter regime.

The temporal and spatial evolution of the impurity density is determined by the impurity flux (2) and can be obtained from solving the set of coupled rate equation for all different ionization states $\mathrm{Z}$ of the impurity species [3]

$$
\frac{d n_{Z}}{d t}+\frac{1}{r} \frac{d}{d r}\left(r \Gamma_{Z}\right)=n_{e}\left(S_{Z-1} n_{Z-1}-S_{Z} n_{Z}+R_{Z+1} n_{Z+1}-R_{Z} n_{Z}\right)
$$


On impurity handling in High Performance Stellarator/Heliotron plasmas

$S_{Z}$ and $R_{Z}$ being the ionization rate and recombination rate coefficients for the corresponding ionization state $Z$, respectively. With the boundary conditions $\mathrm{dn}_{Z} / \mathrm{dr}($ center $)=0$, a constant impurity influx and the assumption of small impurity concentration (tracer approximation) this problem has a stationary solution $\left(\Gamma_{Z}=0\right)$ with a linear relation between impurity source strength at the plasma edge and central impurity concentration in the stationary phase. The core confinement of impurities finally determines the time scale for impurity exhaust.

In a plasma with significant inward term, the impurity concentration rises transiently with a time constant depending on the ratio of convective to diffusive transport. The corresponding increase of impurity radiation is usually the observable indicator for accumulation if changes in the ambient plasma $\left(\mathrm{n}_{\mathrm{e}}, \mathrm{T}_{\mathrm{e}}\right)$ can be excluded. The growing radiation saturates when stationarity has been approached, i.e. when $\Gamma_{Z}=0$ for all $Z$. The convective transport results in a peaking of the total impurity density profile with respect to the density profile of the background ions depending on the ratio $V_{Z} / D_{Z}$ characterizing the relative strength of the impurity pinch. The reason for the peaking is that the additional impurity inward fluxes $\mathrm{n}_{\mathrm{Z}} \mathrm{V}_{\mathrm{Z}}$ have to be compensated by larger diffusive impurity outward fluxes $\mathrm{D}_{Z} \mathrm{n}_{\mathrm{Z}}$ ' due to steeper impurity ion gradients.

Hence, also in plasmas with large $V_{Z} / D_{Z}$, i.e. long impurity core confinement times, stationarity is expected ultimately. The question is now whether the equilibrium level of the impurity radiation losses is low enough in order not to degrade the plasma energy and to initiate a radiative collapse. Since the stationary level is linked to the net impurity influx into the plasma core, as mentioned before, reduction or shielding of the impurity sources in plasmas with long impurity confinement might allow successful sustainment of the plasma at a reasonable radiation level. Such a retention of the impurity influx can be achieved by direct shielding mechanisms via friction or, i.e., by periodic particle exhaust in the SOL as known from ELM activity (edge localized modes). This can be described by an "effective" impurity influx. Examples for these mechanisms are summarized in section 4 .

\section{Observed trends for impurity confinement}

Impurity transport studies were performed at LHD, W7-X and TJ-II, analyzing the temporal and spatial evolution of impurity radiation in response to transient impurity sources. For this purpose short pulses of impurity ions were injected into the plasma either by tracer-encapsulated pellets using titanium as tracer (LHD) or the laser blow-off technique with aluminium (W7-AS) and silicon (TJ-II) as tracer material. In all cases the decay times of the corresponding line emission of the highest ionization states after the pulse were taken as a measure for the impurity confinement time. For low density plasmas the investigations reveal poor impurity confinement in TJ-II and LHD $\left(\mathrm{n}_{\mathrm{e}}<10^{19} \mathrm{~m}^{-3}\right)$ as well as in W7-AS $\left(\mathrm{n}_{\mathrm{e}}<5 \times 10^{19} \mathrm{~m}^{-3}\right)[2,4,5]$. This was attributed to either strong turbulent transport (W7-AS, LHD [6,8]) or the appearance of the electron root at low collisionality (LHD [7]). Consistently, the plasma discharges could be maintained without any accumulation of intrinsic impurities. Additionally, a clear density dependence of the impurity confinement emerges during dedicated density scans in TJ-II, W7-AS and LHD [2,4,5] due to an increasing ratio of inwards convection $\mathrm{V}$ to diffusion coefficient $\mathrm{D}$ in the bulk plasma and thus longer confinement times at higher density as shown in FIG.1 for the three devices. This was partly attributed to a density dependence of D in W7-AS which is consistent with a scaling of the aluminium confinement time $\tau_{\boldsymbol{A l}} \propto \boldsymbol{a}_{\boldsymbol{p}}{ }^{2.4} \cdot \hat{\boldsymbol{n}}_{\boldsymbol{e}}^{1.2} \cdot \boldsymbol{B}^{0.3} / \boldsymbol{P}_{\mathbf{E C R H}}^{0.8}$ (plasma radius $\mathrm{a}_{\mathrm{p}}$, central electron density $\hat{n}_{e}<$ $5 \cdot 10^{19} \mathrm{~m}^{-3}$, magnetic field $\mathrm{B}$, electron cyclotron radiation heating $(\mathrm{ECRH})$ power $\mathrm{P}_{\mathrm{ECRH}}$ ) and a density and density-gradient-dependent inwards convection velocity V in LHD [8]. In LHD, which 
On impurity handling in High Performance Stellarator/Heliotron plasmas

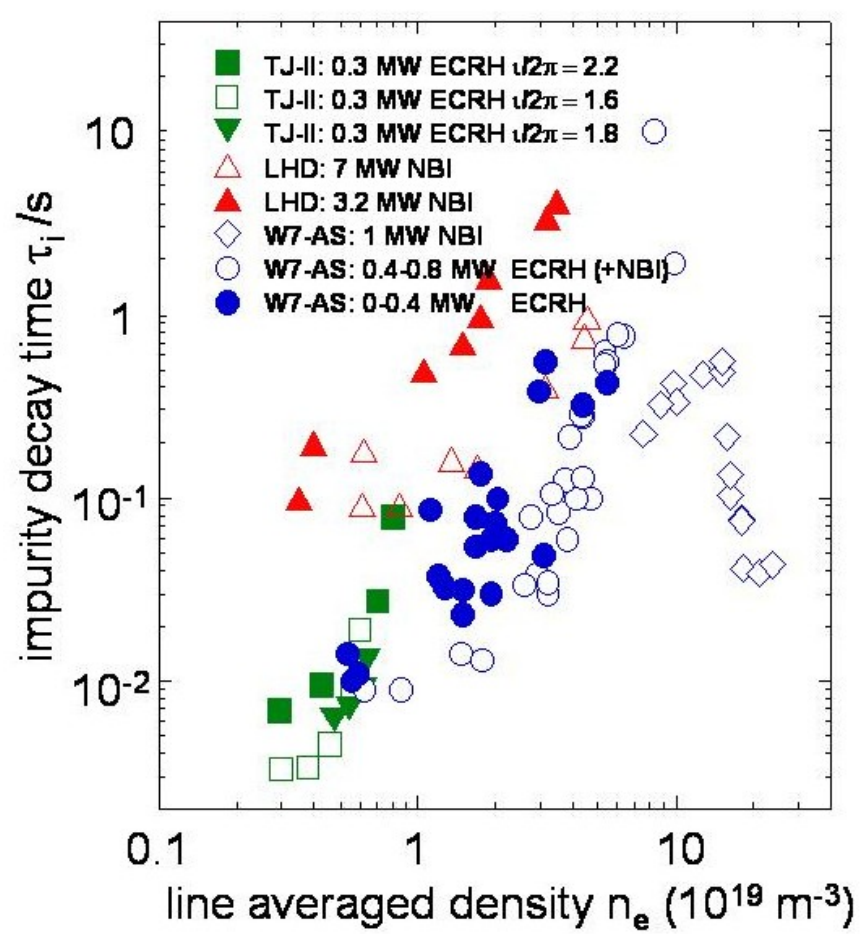

FIG. 1. Impurity confinement time vs. density in TJ-II, LHD, W7-AS. Open blue diamonds contain W7-AS HDH data. The data point at 10s should indicate no decay in W7-AS optimum confinement (OC) plasmas within the pulse duration. has a large flexibility in changing the ambipolar radial electric field $E_{r}$ by controlling the effective ripple and the magnetic topology, the impact of $E_{r}$ on impurity transport was studied [7,9]. Consistently, a radial electric field evolving gradually from positive (outward flow of impurities) to negative (inward flow) values with growing density was found. In addition, a reduction of turbulent transport in both machines at higher density is assumed to account for the density scaling of the impurity behaviour. Although direct evidence of turbulent transport is difficult to obtain, there are some convincing indications pointing to the essential role of anomalous impurity transport, in particular at lower and medium density [2,6]: 1 . The impurity diffusion coefficient D decreases with density (W7-AS) or is independent of density (LHD). This is in contrast to conventional neoclassical scaling, which predicts an increase of $\mathrm{D}$ with density for both cases. Transport simulations at

LHD [6,8] reveal diffusion coefficients being one order of magnitude enhanced over the neoclassical level for low and medium density. Towards high densities experimental and neoclassical D's seem to approach each other. 2. At W7-AS both impurity and energy confinement obey quite similar dependences on global parameters (density, heating power etc.) suggesting common driving mechanisms.

The consequences of the unfavourable impurity confinement at high density manifest themselves in radiation losses that steadily rise throughout the pulse duration by accumulating impurity ions [2,7]. At fractions of radiation power losses larger than $40-50 \%$ of the heating power the plasma energy starts to degrade in W7-AS and the discharge terminates by radiation instability (FIG. 2.). The maximum achievable pulse length becomes therefore a function of the electron density for a given heating power and impurity concentration. The high MHD stability limits do not restrict the operational range in these discharges and are not responsible for the energy degradation. Hence, the radiation losses determine the density limit in W7-AS, which - in contrast to tokamaks - is a soft limit and depends on heating power and impurity concentration. Therefore, both degradation of impurity confinement in the bulk plasma as well as screening of impurity influx at the edge are of equal necessity for successful long-pulse operation. The radiation losses are mainly dominated by VUV radiation from medium ionization states and therefore inversely 
On impurity handling in High Performance Stellarator/Heliotron plasmas

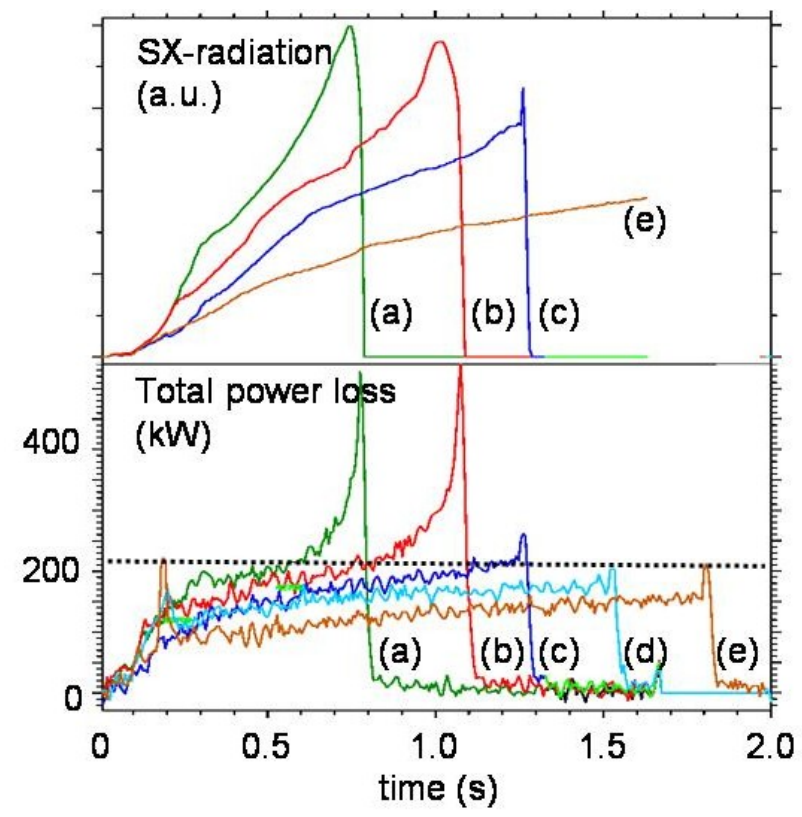

FIG. 2. Impurity radiation in $0.5 \mathrm{MW}$ NBI-heated W7-AS plasmas at constant density of 0.87 ((e,d), \#42721/42714) / 1.05 ((c), \#42709) / 1.15 ((b), \#42706) / $1.25 \cdot 10^{19} \mathrm{~m}^{-3}((a), \# 42698)$. The dashed line indicates $40 \%$ of heating power [10].

scaling with the electron temperature. Consequently, sufficient heating power contributes beneficially to avoid early limitation of the pulse length.

\section{Basic approaches for reduction of impurity radiation losses}

\subsection{Reduction of impurity sources}

Generally, impurity accumulation does not necessarily lead to a radiation collapse if the influx is kept small: for densities $\mathrm{n}_{\mathrm{e}}>5 \times 10^{19} \mathrm{~m}^{-3}$ successive onset of accumulation is observed in W7-AS limiter ECRH discharges due to an overall reduction of $\mathrm{D}$ and increase of $\mathrm{V}_{\text {inward }} / \mathrm{D}$ in particular at the plasma boundary, where the usually peaked profiles of D have their smallest values [2]. Central fluorine radiation continued rising while the fluorine influx was kept constant by an external gas valve in W7-AS. This behaviour could be well simulated by means of a reduced D at higher density, which is assumed to be a consequence of reduced turbulent transport. However, the simulations predict a saturation of the power loss after a time constant of $1.7 \mathrm{~s}$ (longer than the

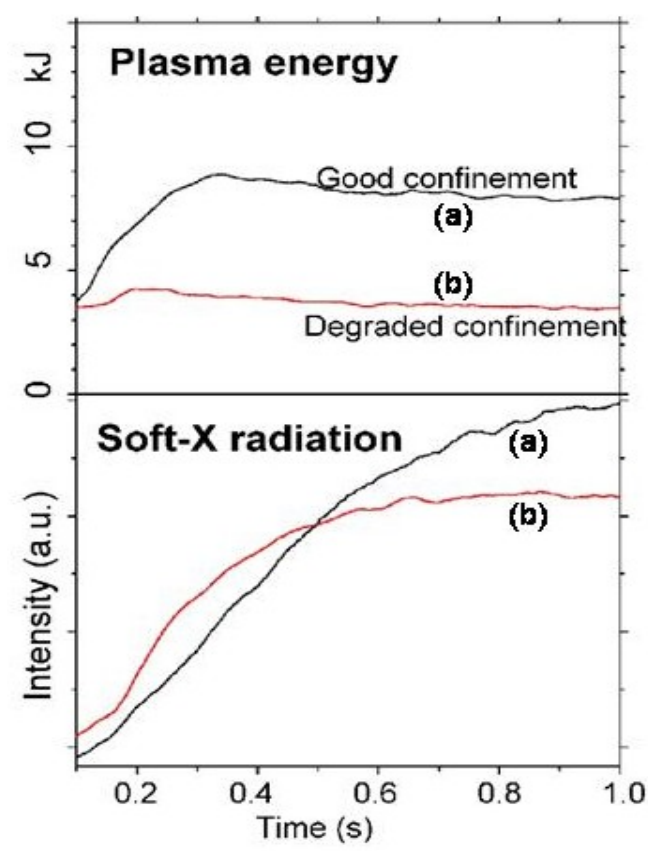
technically limited pulse length of $1 \mathrm{~s}$ ) which is mainly determined by the low edge diffusion coefficient. Extrapolation to $1.7 \mathrm{~s}$ reveals a saturation level for the radiation loss of about $40 \%$ of the heating power in this particular case, which is marginally below the level where degradation of the plasma energy by total radiation losses is expected. The impact of the impurity transport coefficients on the time scale for achieving stationarity is indicated in FIG. 3. where two plasmas with good and slightly deteriorated confinement properties are compared. In case of higher impurity transport (reduced confinement) stationarity is achieved sooner (curves (b)). For

FIG. 3. Evolution of soft-X radiation in a plasma with good (a: \#39226) and deteriorated (b: \#39245) energy and impurity confinement. 
On impurity handling in High Performance Stellarator/Heliotron plasmas

longer confinement times saturation is indicated (curves (a)) but probably not achieved during the pulse duration. Because the final saturation level is directly related to the impurity source strength, an accumulating plasma can nevertheless be maintained stationary if the sources can be kept sufficiently small. This points to the particular necessity to eliminate avoidable or at least to reduce impurity sources from the very beginning of plasma operation by carefully shielding the plasma facing in-vessel components as a first "passive" approach (for more sophisticated scenarios see sec. 5).

\subsection{Utilization of power degradation of impurity confinement}

Utilization of the observed power dependence of the impurity confinement time (see confinement time scaling in section 2 and power degradation of impurity confinement at LHD and W7-AS in FIG. 1.) is a pragmatic tool to exert influence on the impurity concentration. The degradation of confinement at higher ECRH power is a consequence of enhanced D in W7-AS and both, enhanced D and changing V in TJ-II, which might indicate an increased anomalous transport. As demonstrated in W7-AS-discharges with three consecutive ECRH heating power steps performed at five different densities, accumulation can be successfully suppressed by application of sufficient heating power, e.g. $1.2 \mathrm{MW}$ at a central density of $9 \cdot 10^{19} \mathrm{~m}^{-3}$ (FIG. 4.). This corresponds to a volume-averaged power density of approximately $1 \mathrm{MW} / \mathrm{m}^{3}$. The existing restriction of ECRH heating to densities below the cut-off density $\left(1.2 \cdot 10^{20} \mathrm{~m}^{-3}\right.$ for $140 \mathrm{GHz}$ ECRH second harmonic Xmode resonant absorption) can possibly be overcome by new microwave heating scenarios such as Electron-Bernstein Wave heating (EBW). The Electron-Bernstein waves are excited via the O-X-B mode conversion process and extends the operational density range with no upper limit. This heating scenario might pave the way for high density ECRH operation and the utilization of power degradation even at high density [11].

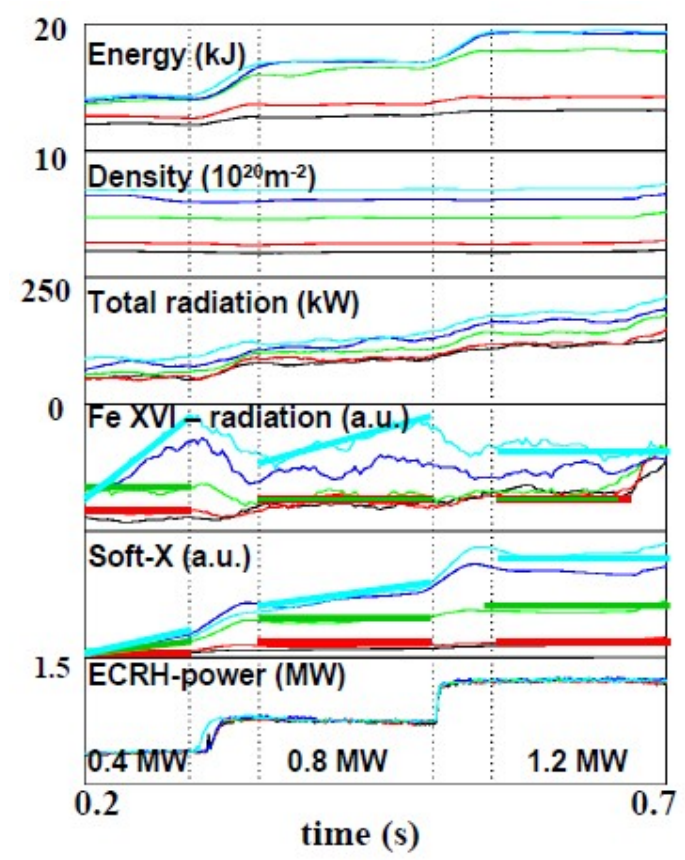

\section{Particular purification features in High performance plasmas}

Numerous improved confinement modes at medium and high density were observed in helical devices, such as e.g. High $\mathrm{T}_{\mathrm{i}}$ (-like) mode at HELIOTRON $\mathrm{E}$ and CHS, edge transport barrier (ETB) and Reheat-mode (improved Lmode) plasmas at CHS, H-mode-like transitions and Super Dense Core plasmas in LHD, and optimum confinement (OC, earlier: $\mathrm{H}-\mathrm{NBI})$ as well as H/HDH-mode plasmas in W7-AS. Some of them are affected by impurity accumulation

FIG. 4. Evolution of impurity radiation in plasmas with different density and heating power (\#43133,43134, 43140, 43143, 43144). Same order of time traces from top to bottom in each subframe. The straight bars in the $4^{\text {th }}$ and $5^{\text {th }}$ subframe should point up the trend of the impurity radiation evolution more clearly $\lceil 21$. 
On impurity handling in High Performance Stellarator/Heliotron plasmas

phenomena which often prevents them from stationary performance - some of them aren't. Nevertheless, the comparison of these scenarios might disclose potential ways out of the dilemma and are discussed below.

\subsection{Impurity influx shielding in the edge/SOL of divertor plasmas}

Both, the helical divertor in LHD and the island divertor in W7-AS (and W7-X) demonstrate favourable impact on the impurity retention $[12,13]$ in the SOL. Generally in both, tokamaks and non-axisymmetric devices, the parallel ion temperature gradient along the separatrix (so-called "upstream" position in the divertor jargon) to the lower temperature in front of the divertor plates ("downstream") causes a competition between downstream directed frictional plasma flow flushing the impurity ions back to the divertor plates and parallel upstream directed impurity flux induced by thermal forces on the impurity ions. The latter depends on the temperature gradient $\left(\nabla \mathrm{T}_{\mathrm{i}}\right)$ and, via the collision frequency, quite sensitively on $\mathrm{T}_{\mathrm{i}}$ ( scaling as $\mathrm{T}_{\mathrm{i}}^{3 / 2}$ ). A reduction of the impurity influx can be achieved by reduction of $\nabla T_{i}$ or the upstream $T_{i}$. When the parallel heat conduction governs the energy transport in the SOL as in tokamaks, there is no direct way to control $\nabla T_{i}$ and the upstream $T_{i}$ independently. A reduction of the downstream $T_{i}$ will cause steepening of the temperature gradient, yielding no net effect of reducing the impurity influx. Nonaxisymmetric divertors based on islands, however, have the potential to decrease $\nabla \mathrm{T}_{\mathrm{i}}$ and the upstream $T_{i}$ simultaneously by additional cross-field heat transport through the islands which grows with density and acts like a supplementary bypass between up- and downstream positions. This causes a strong reduction of the parallel conductive heat flux (scaling as $\mathrm{T}_{\mathrm{i}}^{5 / 2}$ ) and finally of the thermal impurity influx. As a consequence, the impurity density at the separatrix as well as the impurity influx into the core region [12] is decreased. In detail, this might differ depending on the real geometry of the divertor structure.

This impurity screening mechanism was well simulated by the EMC3/EIRENE code and experimentally demonstrated in the SOL of W7-AS during the HDH-mode [12] and also in the stochastic (magnetic) edge and surface layer of LHD [13]. With the onset of the HDH mode in W7-AS at central densities of $1.5-2.2 \cdot 10^{20} \mathrm{~m}^{-3}\left(\mathrm{P}_{\mathrm{NBI}}=1-3.5 \mathrm{MW}\right)$ and edge densities above $1-2 \cdot 10^{19} \mathrm{~m}^{-3}$ the impurity influx is reduced remarkably and enables stationary operation at high density up to $4 \cdot 10^{20} \mathrm{~m}^{-3}$ without accumulation - which was not possible before. An essential ingredient for achieving high edge density (and low edge temperature) was the use of strong gas puffing. This transiently forces the formation of a broad H-mode like density profile with steep edge gradients. Large negative radial electric fields (thermodynamic force $\mathrm{E}_{\mathrm{r}} / \mathrm{T}_{\mathrm{i}}$ ) at the plasma boundary, suppressed fluctuation activity and the resulting reduced edge transport might be responsible for the self-consistent stabilization of the HDH-mode.

In LHD, the trend for accumulation in long-pulse experiments $\left(\mathrm{P}_{\mathrm{NBI}}=1.5 \mathrm{MW}\right)$ at medium density (accumulation window $1.0-2.7 \cdot 10^{19} \mathrm{~m}^{-3}$ ) was stopped beyond a relatively low (compared to W7-AS in HDH-mode) power dependent line averaged density of $2.7 \cdot 10^{19} \mathrm{~m}^{-3}$ [7]. However, the impurity core confinement time (impurity pellet injection experiments) still remains long (!) beyond the accumulation window, indicating that the onset of purification is not exclusively determined by the core confinement: the injection experiments are sensitive to the core confinement whereas the global intrinsic impurity behaviour might be dominated by edge shielding processes as well, which is no contradiction. Likewise, the Super Dense Core (SDC) plasmas with internal diffusion barrier $\left(\mathrm{n}_{\mathrm{e}}(0)=5-6 \cdot 10^{20} \mathrm{~m}^{-3}, \mathrm{~T}_{\mathrm{e}}(0)=660-850 \mathrm{eV}\right)$ obtained in LHD by frequent pellet injection in the presence of a helical divertor or the local island divertor (LID) plasmas presumably do not suffer severely from enhanced radiation $[14,15]$, although impurity 
On impurity handling in High Performance Stellarator/Heliotron plasmas

accumulation and long impurity confinement are indicated [16]. In both cases, consequently, a very effective shielding of the impurity influx in LHD has to be suggested which can most likely be assigned to the same intrinsic edge screening mechanism discussed above, so that the observations at LHD and W7-AS seem to be consistently explained by the same screening mechanism.

Moreover, in the long-pulse experiments at LHD even with densities inside the accumulation window impurity control is also successively demonstrated during generation of an $\mathrm{n} / \mathrm{m}=1 / 1$ magnetic island near the plasma edge by means of external perturbation coils. After application of the perturbation field the initially rising central radiation decreases remarkably on a large time scale, the radiation profile centrally dropping from peaked to hollow. This may be interpreted as the consequence of a significantly reduced impurity influx [7], the exact mechanism still being under discussion. During additional operation of the LID in the magnetic island configuration a positive radial electric field with a strong shear was produced at the plasma edge by electron loss to the LID, triggering an internal transport barrier (ITB) and reducing the impurity influx [9]. All these phenomena stress the relevance of impurity edge transport for successful prevention of excessive radiation losses and stationary performance - in addition to the core confinement.

\subsection{Degradation of impurity core confinement at high density}

Simultaneously with the onset of the screening of impurity influx into the W7-AS HDH plasma, also the impurity core confinement drops dramatically at high density (see FIG. 1.) in contrast to LHD. With the onset of the HDH mode the density profile suddenly flattens whereas the temperature profiles remain peaked. Both improved energy and degraded impurity core confinement (measured by impurity injection technique) could be demonstrated [2], which is favorable for fusion devices but difficult to achieve simultaneously. At these high densities impurity transport simulations for HDH reveal nearly neoclassical character. One of the reasons for the sudden drop of impurity confinement is a reduced inward core convection which is neoclassically consistent with the flattening of the density profiles. However, neoclassical simulations additionally predict large inwards directed impurity fluxes over the range of the strong edge density gradients during HDH. This was experimentally substantiated by derivation of a narrow layer of strong negative radial electric field $\mathrm{E}_{\mathrm{r}}$ from the edge pressure profile, driving the inwards flux. The existence of this layer is predicted to cause long impurity confinement times in contrast to the observations. Finally, the introduction of an artificially enhanced impurity edge diffusion coefficient, representing some still unknown transport mechanisms locally counteracting the impurity inwards convection, was necessary to match the extremely small observed impurity confinement times (down to $10 \mathrm{~ms}$ ). Code calculations show that changes of this anomalous edge diffusion coefficient and hence changes in the two balancing edge transport processes have substantial impact on the global impurity confinement - ranging from extremely short to nearly infinite impurity confinement times. This additionally underlines the importance of edge/SOL transport for the global impurity confinement. An experimental indication for this assumption is the observation, that in spite of similar density and temperature profiles the impurity confinement in the ELM-free H-mode $\left(\mathrm{H}^{*}\right)$ is extremely long compared to the short confinement times in HDH. Surprisingly, the achievement of HDH properties also in high- $\beta$ limiter plasmas indicates that a divertor configuration might not necessarily be a prerequisite. 
On impurity handling in High Performance Stellarator/Heliotron plasmas

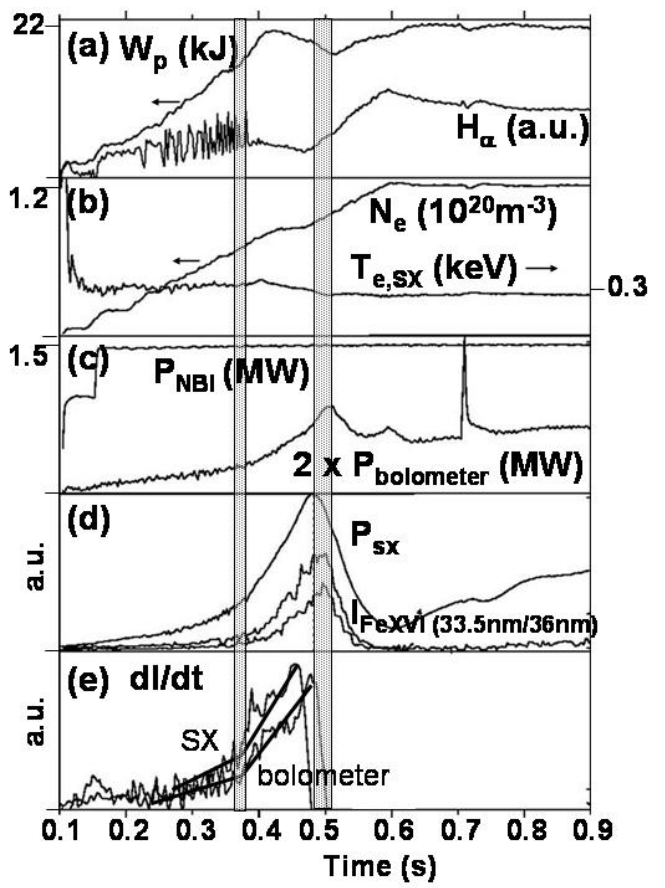

FIG. 5. W7-AS discharge \#56156 with transition from $\mathrm{H}$-mode to $\mathrm{H}^{*}$-mode (around 0.37s) and then to HDH-mode (around 0.5s).

(a) Plasma energy $W_{p}, H_{\alpha}$, (b) average density $N_{e}$, electron temperature derived from $S X$ radiation $T_{e, S X}$, (c) NBI and total radiation power, (d) SX-radiation power and FeXVI line radiation, (e) derivative of bolometer and $S X$ radiation power.

\subsection{Impact of Edge Localized Modes (ELMs)}

At W7-AS, two improved confinement modes exist at medium density (approx. $8 \cdot 10^{19} \mathrm{~m}^{-3}$ ) which do not benefit from impurity screening inferred by high SOL collisionality as described in section 5.1 and have to utilize other mechanisms: 1. The normal H-mode has flat core density profiles and steep edge gradients similar to $\mathrm{HDH}$, but insufficiently high edge density and low edge temperature needed for effective friction induced edge screening. 2. The standard ion-root optimum confinement regime (OC, similar to High $\mathrm{T}_{\mathrm{i}}$ modes in CHS and HELIOTRON $\mathrm{E}$ [17]) with peaked density profiles, inward shifted density gradients (often larger than in ELM-free $\mathrm{H}$-modes $\left(\mathrm{H}^{*}\right)$ ), low recycling and broad ion temperature profiles. In the normal $\mathrm{H}$-mode the already long impurity confinement inferred by the density scaling is even enhanced, and the additional absence of ELMs $\left(\mathrm{H}^{*}\right)$ leads to an exponential increase of radiation provoking an abrupt termination of the pulse. If the density is ramped up quickly enough to more than $1.5 \cdot 10^{20} \mathrm{~m}^{-3}$, again stationary operation without accumulation in the HDH-mode becomes possible (FIG. 5.). Nevertheless, the residence time in the $\mathrm{H}^{*}$-mode must be kept short in order to avoid enrichment of the core plasma by impurity ions and thus the onset of energy degradation by enhanced radiation before reaching the $\mathrm{HDH}$ regime. The NBI-heated OC-regime is also afflicted by accumulation but cannot be treated by rigid procedures such as strong impurity puffing as discussed in section 5.1. The OC-regime is the result of a complex bifurcation process evolving gradually on a particle transport time scale under defined conditions for NBI heating power, density, recycling and additional ECRH-power for density control. The highest ion temperatures and longest energy confinement times at W7-AS were achieved in this regime, which was found to behave almost neoclassically.

In both modes ELMs develop with a large variation in their frequency in the H-mode and often a grassy character in OC plasmas, which might be utilized to prevent accumulation. The beneficial impact of ELMs on impurity concentration in H-modes but also their drawbacks for fusion reactors with respect to excessive power loads onto plasma facing in-vessel components in case of large single ELMs are permanent subjects of investigations at tokamaks. During the last years, at TJ-II, LHD and W7-AS extensive studies on the nature of ELMs or ELM-like bursts in Stellarators/Heliotrons $[18,19,20,21]$ were performed. With respect to the general trend for improved core impurity confinement at high density, however, it is worthwhile to elucidate also the role of ELMs as possible candidates for plasma purification in helical systems. FIG. 6. shows 
On impurity handling in High Performance Stellarator/Heliotron plasmas

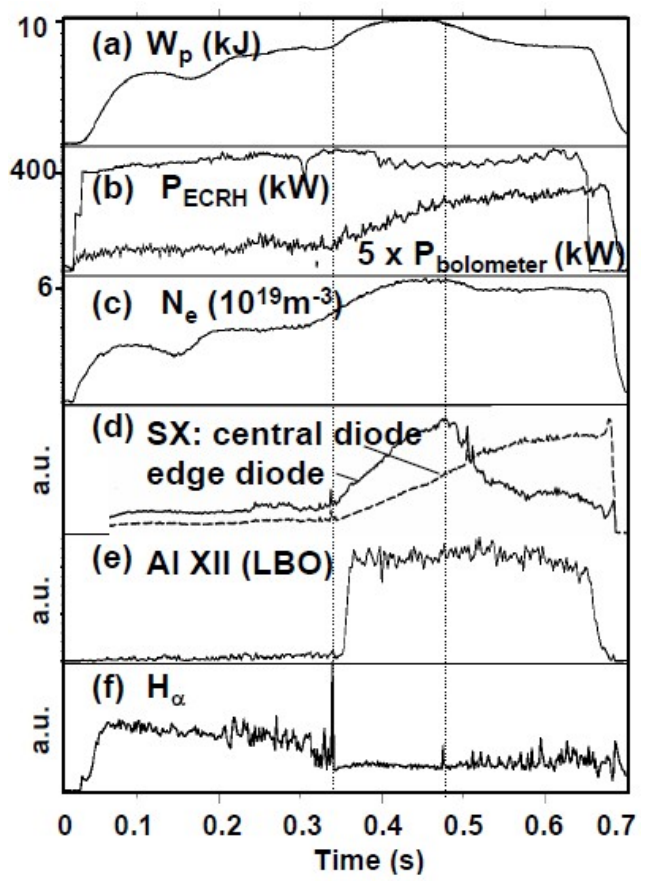

FIG. 6. W7-AS discharge \#21142 with $L-H$ transition at 0.34s. (a) Plasma energy $W_{p}$, (b) ECRH heating and total radiation power, (c) average density, (d) radiation of central and edge (inside ELM pivot point) SX-diode, (e) Al XII radiation after Al laser blow-offat $0.35 \mathrm{~s},(f) H_{\alpha}$

the onset of increasing core radiation (central channel of Soft-X-camera) starting with the formation of the $\mathrm{H}^{*}$-mode and a flattening during the onset of frequent ELM activity. The time trace of the Al XII emission after a short aluminium laser blow-off injection reveals nearly no significant decay even in the phase with ELMs, indicating still long impurity confinement in the presence of saturating bulk radiation. Consistent to the experiences from tokamaks, these are indications that frequent ELMs have impact on the net impurity influx into the core plasma and can principally be helpful in the suppression of accumulation in both high performance scenarios. Large single ELMs have significant impact on the edge radiation profiles but reveal less effective impurity shielding than frequent ELM activity of moderate amplitude. The challenging task is, of course, to identify windows in the configurational space in oder to control the appearance, prevention or mitigation of ELMs.

\section{Summary}

Stellarators/Heliotrons have the intrinsic potential for continuous operation and focus on the achievement of high performance plasmas which can be maintained stationary. However, theoretical predictions basically forecast accumulative impurity behaviour in non-axisymmetric devices in the standard ion root regime. Moreover, experimental findings reveal some unfavorable density dependence of the impurity confinement when going to dense or improved confinement plasmas. As a consequence, long impurity confinement times for the core plasma at higher density are found at several machines.

Nevertheless, various operational scenarios were identified or already explored in present generation Stellarators/Heliotrons which provide approaches to effectively control the impurity influx: (a) careful reduction of impurity sources, (b) application of heating power degradation of impurity confinement, or establishing favourable edge transport mechanisms such as (c) frequent ELM activity, (d) impurity screening at high edge collisionality by friction force, (e) introduction of $n / m=1 / 1$ magnetic islands and positive radial electric fields at the plasma edge. The latter are specific advantages of non-axisymmetric devices and can be sufficiently understood from classical theory.

It was demonstrated at LHD that in the presence of effective edge screening mechanisms steady state operation seems possible at a reasonable radiation level - in spite of long impurity confinement time at higher densities. Additionally to the reduction of impurity influx a 
On impurity handling in High Performance Stellarator/Heliotron plasmas

degradation of impurity core confinement was obtained in W7-AS HDH-plasmas, obviously depending also sensitively on transport processes at the plasma edge. This stresses the importance and necessity to include three-dimensional effects additionally to the conventionally used axisymmetric approximations of neoclassical theory in impurity transport modelling - not only to understand current observations but also to explore further operation regimes.

Both the role of turbulent transport with respect to power degradation of impurity confinement and the edge transport processes in HDH need further investigations for better understanding. The effect of ELMs on the reduction of the net impurity influx was shown but their controlled applicability needs further exploration. From the inter-machine comparison it can be finally concluded, that - in spite of the primary unfavourable density scaling of the global impurity confinement - effective screening of impurity seems possible in Stellarators/Heliotrons providing stationary radiation power loss conditions at an acceptable level.

\section{References}

[1] Maassberg H., Beidler C D and Simmet E E 1999 Density control problems in large stellarators with neoclassical transport Plasma Physics and Controlled Fusion 411135

[2] Burhenn R et al 2004 Impurity transport studies in the Wendelstein 7-AS stellarator Fusion Science and Technology 46115

[3] Isler R C 1984 Impurities in tokamaks Nucl. Fus. 24,1599

[4] Hidalgo C et al 2005 Overview of TJ-II experiments Nucl. Fus. 45, S266

[5] Tamura $\mathrm{N}$ et al 2003 Impurity transport studies by means of tracer-encapsulated solid pellet injection in neutral beam heated plasmas on LHD Plasma Physics and Controlled Fusion 45(1)27

[6] Morita S et al 2006 Spectroscopic studies on impurity transport of core and edge plasmas in LHD Plasma Science \& Technology 8(1) 55

[7] Nakamura Y et al 2003 Plasma performance and impurity behaviour in long pulse discharges on LHD Nucl. Fus. 43219

[8] Nozato H, Morita S, Goto M, Takase Y, Ejiri A, Amano T, Tanaka K, Inagaki S and LHD experimental group 2006 Phys. Plasmas 13(5), 092502

[9] Ida K.et al 2005 Control of the radial electric field shear by modification of the magnetic field configuration in LHD Nucl. Fus. 45391

[10] Giannone L et al 2000 Physics of the density limit in the W7-AS stellarator Plasma Physics and Controlled Fusion 42603

[11] Laqua H P 2007 Electron Bernstein wave heating and diagnostic Plasma Physics and Controlled Fusion 49 R1

[12] Feng Y., Sardei F, Grigull P, McCormick K, Kisslinger J and Reiter D 2006 Physics of island divertors as highlighted by the example of W7-AS Nucl. Fus. 46(8) 807

[13] Kobayashi M, Feng Y, Masuzaki S, Morisaki N, Yamada H, Komori A, Motojima O and the LHD experimental group 2008 Contrib. Plasma Phys. 48(1-3) 255

[14] Yamada H et al 2007 Characterization and operational regime of high density plasmas with internal diffusion barrier observed in the Large Helical Device Plasma Physics and Controlled Fusion 49

[15] Morisaki T et al 2007 Superdense core mode in the Large Helical Device with an internal diffusion barrier Physics of Plasmas 14 056113-1

[16] Tamura N et al 2007 Tracer impurity transport incide an IDB plasma of LHD Proc. Joint Conf. of 17th Int. Toki Conf. and $16^{\text {th }}$ Int. Stellarator/Heliotron Workshop, Toki, Japan

[17] Ida K et al. 1996 High ion temperatura mode in Heliotron-E Phys. Rev. Lett 76 (8) 1268

[18] Weller A, Anton M, Geiger J, Hirsch M, Jaenicke R, Werner A, W7-AS Team, Nuehrenberg C, 
On impurity handling in High Performance Stellarator/Heliotron plasmas

Sallander E and Spong D A 2001 Phys. Plasmas 8931

[19] Jimenez J A., De la Luna E, Garcia-Cortes I and Shchepetov S V 2006 Localized electromagnetic modes in MHD stable regime of the TJ-II Heliac Plasma Physics and Controlled Fusion 48515

[20] Morita, S et al 2007 H-mode-like transition an ELM-like bursts in LHD with thick ergodic layer Nucl. Fus. 471033

[21] Hirsch, M et al 2009 Major results from the stellarator Wendelstein 7-AS PPCF 50(5) 053001 